\title{
Effets de la supplémentation de l'aliment en poudre des feuilles de goyavier (Psidium guajava) sur les performances de croissance des porcelets
}

\author{
François DJITIE KOUATCHO ${ }^{*}$, Victor M. KUATE SILATCHOM ${ }^{2}$ et \\ Ferdinand NGOULA ${ }^{2}$ \\ ${ }^{1}$ Département des Sciences Biologiques, Faculté des Sciences, Département de Génie Chimique, EGCIM, \\ Université de Ngaoundéré, Cameroun. \\ ${ }^{2}$ Département des Productions Animales, Faculté d'Agronomie et des Sciences Agricoles, Université de \\ Dschang, Cameroun. \\ *Auteur correspondant ; E-mail: franckdjitie@gmail.com; Tel (237)694802786 ; BP : 454 Ngaoundéré, \\ Cameroun.
}

Received: 27-11-2020

Accepted: 19-03-2021

Published: 30-04-2021

\section{RESUME}

Dans le but de contribuer à une meilleure connaissance des effets des feuilles de goyavier sur la croissance en élevage porcin, une étude a été menée sur les effets de la supplémentation de l'aliment en poudre des feuilles de goyavier (Psiduim guajava) sur la croissance des porcelets hybrides (Large White x Landrace). A cet effet, 24 porcelets de 65 jours d'âge et de poids moyen de 10,83 $\pm 0,26 \mathrm{~kg}$ ont été répartis en quatre lots de 6 porcelets ( 03 mâles et 03 femelles) chacun. A chaque lot choisi au hasard a été attribué par jour et pendant 30 jours consécutifs une ration supplémentée avec $0 ; 0,5 ; 1$ et $1,5 \%$ de poudre des feuilles de goyavier. Les performances de croissance ont été étudiées. La consommation alimentaire et le poids vif ont été comparables entre tous les traitements. Le gain de poids, le gain moyen quotidien et l'efficacité alimentaire ont été significativement $(\mathrm{P}<0,05)$ plus élevés avec la ration contenant $1,5 \%$ de poudre de feuilles de goyavier comparé à la ration contenant seulement $1 \%$ de cette poudre. L'indice de consommation et le coût de production ont été relativement plus faibles chez les porcelets ayant reçu 1,5\% de poudre des feuilles de goyavier comparés à ceux des autres traitements. Il a été conclu que la supplémentation de l'aliment au taux de 1,5\% de la poudre des feuilles de goyavier améliore les performances de croissance des porcelets.

(C) 2021 International Formulae Group. All rights reserved.

Mots clés : Psidium guajava, enrichissement, porcelet, croissance, coût de production.

\section{Effect of feed supplementation with dry powder of guava leaves (Psidium guajava) on growth performances of cross breed piglets}

\begin{abstract}
In order to contribute to a better understanding of the effects of guava leaves on growth in pig farming, a study has been conducted on the effects of supplementation of guava leaves powder feed (Psiduim guajava) on the growth of hybrid piglets (Large White $\mathrm{x}$ Landrace). For this purpose, 24 piglets of 65 days of age and an
\end{abstract}


average weight of $10.83 \pm 0.26 \mathrm{~kg}$ were distributed in four batches of 6 piglets each. To each group choosing randomly, diets supplemented with $0 ; 0,5 ; 1$ and 1,5\% of guava leaves powder was assigned daily for thirty consecutive days. Growth performances characteristics were studied. Feed intake and live weight were comparable between all treatments. The weight gain, daily average gain and feed efficiency were significantly higher with the diet containing 1,5\% guava leaves powder as compared to the one with $1 \%$ of that powder. Feed efficiency and the cost of production were non-significantly lower in piglet haven received diet containing $1,5 \%$ of guava leaves powder compared to other treatments. It can there be concluded that food supplementation by $1,5 \%$ with guava leave powder improve growth performances of piglets.

(C) 2021 International Formulae Group. All rights reserved.

Keywords: Psidium guajava, supplementation, piglet, growth performances, production's cost.

\section{INTRODUCTION}

Malgré les fortes potentialités dont dispose le Cameroun pour accroître les productions animales, celles-ci restent faibles (Motsa'a et al., 2019) et toute tentative d'amélioration se heurte à plusieurs contraintes qui réduisent de manière considérable la croissance des animaux. Pour faire face aux contraintes alimentaires et sanitaires, les producteurs utilisent les substances de synthèse telles que les antibiotiques et les hormones pour augmenter les prises alimentaires, optimiser l'utilisation alimentaire, augmenter les performances de croissance, prévenir les maladies (Nikolova et al., 2010), améliorer les conditions sanitaires des animaux et accroître la productivité des élevages en réduisant les coûts de production (Lessard, 2004).

Malgré leurs multiples avantages, ces molécules de synthèse sont coûteuses, et ont souvent des effets secondaires aussi bien sur les animaux que sur les humains (Lessard, 2004). Ceci a poussé de nombreux pays à interdire leur utilisation ramenant ainsi les éleveurs à la situation de départ.

Parmi les alternatives aux composés de synthèses figurent les extraits de plantes. En effet, les tests phytochimiques ont montré que les extraits de plantes possèdent des composés variés tels que : les phénols, les vitamines, les minéraux, (Nwangwa et al., 2007 ; Machebe et al., 2011) qui peuvent stimuler la croissance et améliorer la reproduction des animaux (Nwangwa et al., 2007 ; Machebe et al., 2011 ; Mweugang et al., 2016). Leurs activités sont très variées : œstrogéniques, anti-infectieuses, antifongiques, antibactériennes, antiparasitaires, antimutagènes, aphrodisiaques, antioxydantes etc... (Chen et Yen, 2007).
Psidium guajava fait partie de ces nombreuses plantes utilisées aussi bien en ethnomédecine vétérinaire qu'humaine. Cette plante originaire d'Amérique du Sud appartient à la famille des Myrtaceae et au genre Psidium. Elle pousse également dans les tropiques. Sa hauteur est comprise entre 3 et 6 mètres (Delesalle, 2016) et toutes les parties de la plante sont utilisées en médecine traditionnelle pour lutter contre les maladies gastrointestinales et respiratoires, le diabète, les cancers etc. (Oh et al., 2005). Guessan-Irié et al. (2012) ont montré que $P$. guajava possède plusieurs autres propriétés telles que les propriétés antiseptiques, antibactériennes et antidiarrhétiques. Psidium guajava possède de nombreux composés entre autres (flavonoides, tanins, et phenols...). L'action combinée de ces différents principes actifs agissant en synergie protégerait mieux l'organisme (Pousset, 1989). Robineau-Germosén et al. (1999) ont montré que l'extrait aqueux des feuilles de Psidium guajava a des effets positifs sur de nombreux micro-organismes en particulier Escherichia coli, Pseudomonas aeruginosa, Sarcina lutea, etc. (Robineau-Germosén et al., 1999).

Les travaux relatifs aux effets bénéfiques des feuilles de goyavier chez les animaux d'élevage (Zaminur et al., 2013), sont rares notamment en ce qui concerne les effets des différentes préparations de cette plante sur les performances de croissance des animaux. C'est dans cette logique que ce travail a été initié, avec pour objectif de contribuer à une meilleure connaissance des effets des différentes préparations de la plante sur la croissance des animaux d'élevage, en vue d'accroître le disponible en protéines animales. Plus spécifiquement il s'agissait d'évaluer chez les porcelets hybrides (Landrace $\mathrm{x}$ Large 
White) l'effet de la supplémentation de l'aliment en poudre de feuilles de goyavier (Psidium guajava) sur les performances de croissance et les coûts de production du $\mathrm{kg}$ de poids vif.

\section{MATERIEL ET METHODES \\ Période et zone d'étude}

L'étude a été réalisée en zone forestière à pluviométrie bimodale; précisément à la ferme NGV-Traco Cameroun du village Kollo dans la région du Littoral. La ferme est située à $4^{0} 12^{\prime}$ Latitude Nord et à $9^{\circ} 26^{\prime}$ Longitude Est et à une Altitude de $13 \mathrm{~m}$. La température moyenne annuelle est de $27{ }^{\circ} \mathrm{C}$ et les précipitations sont en moyenne de 2402,8 $\mathrm{mm} / \mathrm{an}$. Les sols sont ferralitiques, de couleurs jaune, acides et fortement lessivés par les précipitations importantes. Ils sont très sablonneux mais aussi très fertiles.

\section{Matériel animal et prophylaxie}

Vingt-quatre (24) porcelets (12 mâles et 12 femelles) hybrides Large White x Landrace âgés en moyenne de 65 jours, issus de la ferme NGV-Traco Cameroun et de poids moyen de $10,83 \pm 0,26 \mathrm{~kg}$ ont été utilisés.

Avant le début de l'essai, tous les animaux ont reçu un traitement préventif contre la gale, les parasites externes et les vers intestinaux à base de l'Ivermectine. L'antibiotique (oxythétracycline) et des vitamines ont été administrés respectivement pour prévenir les maladies bactériennes et réduire le stress du sevrage. Tous ces traitements préventifs ont eu lieu le jour du sevrage. La lutte contre les mouches et les bactéries a porté sur l'utilisation des insecticides, le crésyl et le virunet deux fois par semaine. Les loges, les abreuvoirs et les animaux étaient lavés tous les matins, tout comme le renouvellement de la solution désinfectante (le pédiluve) à l'entrée du bâtiment, et l'accès au bâtiment d'élevage était strictement interdit aux visiteurs.

\section{Logement et équipements}

La porcherie était construite en matériaux définitifs. Les longueurs étaient constituées d'un mur en dur d'une hauteur de $1,15 \mathrm{~m}$ et d'une grille en fer de $1,50 \mathrm{~m}$ de hauteur. Les largeurs étaient entièrement fermées avec une porte en fer de chaque côté. Le bâtiment était entièrement recouvert par un toit en aluminium et le sol dallé. Dans chaque loge se trouve un robinet d'eau utilisé pour nettoyer les loges et laver les animaux. Six porcelets ( 3 mâles et 3 femelles) étaient logés dans une salle de mise-bas de superficie de 4,70 $\mathrm{m}^{2}$. Ces loges étaient équipées des mangeoires en bois fixé au sol d'une capacité de $100 \mathrm{~L}$ et d'abreuvoir en plastique de $10 \mathrm{~L}$.

\section{Alimentation}

Pendant toute la période de l'essai, les porcelets recevaient à volonté de l'eau potable et un aliment complet, dont la composition et les caractéristiques chimiques calculées sont consignées dans le Tableau 1.

\section{Matériel végétal}

Les jeunes feuilles de goyavier (Psiduim guajava) (Photo 1) ont été récoltées pendant la saison sèche entre 6 heures et 7 heures 30 minutes du matin, au sein du campus de l'université de Dschang puis elles ont été lavées, séchées à l'ombre à température ambiante (entre 25 et $32{ }^{\circ} \mathrm{C}$ ), puis broyées (Photo 2) à l'aide d'une machine à maille fine.

\section{Dispositif expérimental et conduite de l'essai}

Dans l'optique de tester l'effet de la poudre des feuilles de goyavier sur quelques paramètres de croissance chez les porcelets, un effectif de 24 animaux de poids $10,83 \pm 0,26 \mathrm{~kg}$ ) a été utilisé. Avant le démarrage de l'essai, un temps d'adaptation de 3 semaines a été observé : 7 jours pour réduire le stress dû au sevrage et 14 jours pour s'habituer aux nouvelles loges expérimentales. Au début de l'essai, les animaux ont été pesés, identifiés et répartis-en 4 lots de 6 porcelets ( 3 mâles et 3 femelles) chacun comparable sur la base de leur poids vif et du sexe. Ces animaux recevaient quotidiennement 4 rations $\mathrm{T} 1, \mathrm{~T} 2$, $\mathrm{T} 3$ et $\mathrm{T} 4$ comportant respectivement $0,0 \%$, $0,5 \%, 1 \%$ et $1,5 \%$ d'additif de poudre de feuilles de goyavier. Les porcelets ont été pesés à jeûne toutes les fins de semaine à l'aide d'une balance à ressort de capacité $100 \mathrm{~kg}$ et de précision $1 \mathrm{~kg}$. L'aliment et l'eau étaient distribués à volonté pendant les 30 jours de l'essai. 


\section{Paramètres étudiés et collecte des données}

La consommation alimentaire et le poids vif à jeûne ont été collectés tous les sept jours sur toute la durée de l'essai à l'aide d'une balance Capacité de $100 \mathrm{~kg}$ et de précision de $1 \mathrm{~kg}$. Les différents paramètres étudiés ont été, la consommation alimentaire, le poids vif, le gain moyen quotidien, l'indice de consommation, l'efficacité alimentaire et le coût de production du $\mathrm{kg}$ de poids. Le gain de poids hebdomadaire a été déterminé en faisant la différence entre deux poids hebdomadaires moyens consécutifs. L'indice de consommation a été obtenu en faisant pour la même période, le rapport entre la consommation alimentaire et le gain de poids. L'efficacité alimentaire était obtenue en faisant le ratio entre le gain de poids et la consommation alimentaire.

Le prix de revient de différentes rations a été calculé en se servant du prix des ingrédients pratiqués sur le marché. Celui du $\mathrm{kg}$ de la poudre des feuilles de goyavier a été estimé à 400 FCFA en prenant en compte les frais relatifs à la récolte, transport, lavage, séchage à l'ombre et le broyage. Le coût du kg de poids vif a été calculé en multipliant l'indice de consommation moyen par le prix de revient du kg d'aliment correspondant.

\section{Analyses statistiques}

Les données obtenues ont été soumises à l'analyse de la variance à deux facteurs selon le dispositif complètement aléatoire pour tester l'effet du niveau de la poudre des feuilles de goyavier sur les paramètres de croissance étudiés chez les porcelets, ainsi que le coût de production du $\mathrm{kg}$ de poids vif. Le test de Duncan au seuil de 5\% a permis de séparer les moyennes lorsqu'il y avait des différences significatives. Les résultats ont été exprimés sous la forme de moyenne \pm écart type. Le logiciel SPSS version 20.0 a été utilisé pour les analyses.

Tableau 1 : Composition centésimale et caractéristique chimique de l'aliment.

\begin{tabular}{lc}
\hline Ingrédients & Quantité (kg) \\
\hline Maïs & 67 \\
Remoulage & 6 \\
Tourteau de Soja & 22 \\
Farine de poisson & 2 \\
Farine d'os & 2 \\
Sel Iodé & 0,5 \\
Premix & 0,5 \\
\hline Total & 100 \\
\hline Caractéristiques calculées de la ration & \\
\hline & 364 \\
Energie digestible (kcal/kg MS) & 4 \\
Protéine brute (\% MS) & 18,5 \\
Energie/Protéine & 197 \\
Calcium (\% MS) & 0,82 \\
Phosphore (\% MS) & 0,55 \\
Calcium/Phosphore & 1,47 \\
Lysine (\% MS) & 1.10 \\
Méthionine (\% MS) & 0,36 \\
Lysine/Méthionine & 3,02 \\
Cellulose brute (\% MS) & 2,53 \\
Sodium (\% MS) & 0,23 \\
\hline
\end{tabular}




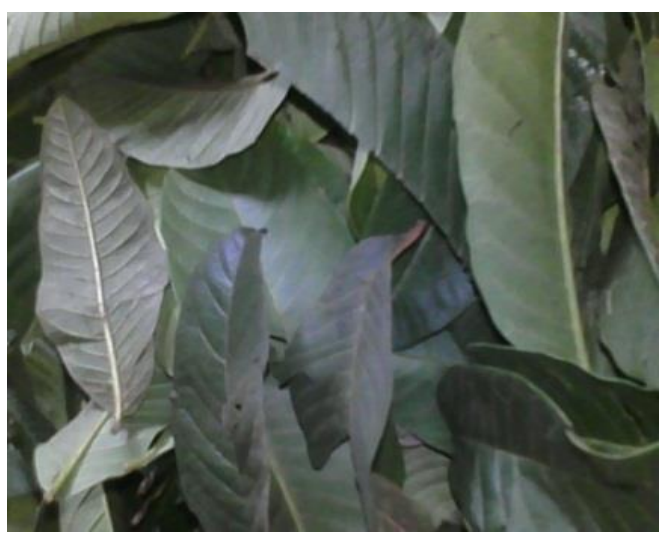

Photo 1 : Jeunes feuilles de goyavier.

\section{RESULTATS}

Effet de la supplémentation de l'aliment en poudre des feuilles de goyavier ( $P$. guajava) et du sexe sur les performances de croissance et le coût de production chez les porcelets

Les effets de la supplémentation de l'aliment en poudre des feuilles de goyavier $(P$. guajava) sur les paramètres de croissance et le coût de production chez les porcelets sont résumés dans le Tableau 2 et illustrés par les Figures 1 à 7 .

\section{Consommation alimentaire des porcelets}

L'évolution hebdomadaire de la consommation alimentaire chez les porcelets est illustrée par la Figure 1. De manière générale, la consommation alimentaire a augmenté du début à la fin de l'essai quel que soit le traitement. Cette augmentation a été plus importante chez les animaux du lot témoin par rapport à ceux traités à la poudre des feuilles de goyavier, de la première à la troisième semaine de l'essai. L'inverse a été observé de la troisième à la quatrième semaine de l'essai. Au terme de l'essai, aucune différence significative $(\mathrm{P}>0,05)$ n'a été observée entre les traitements.

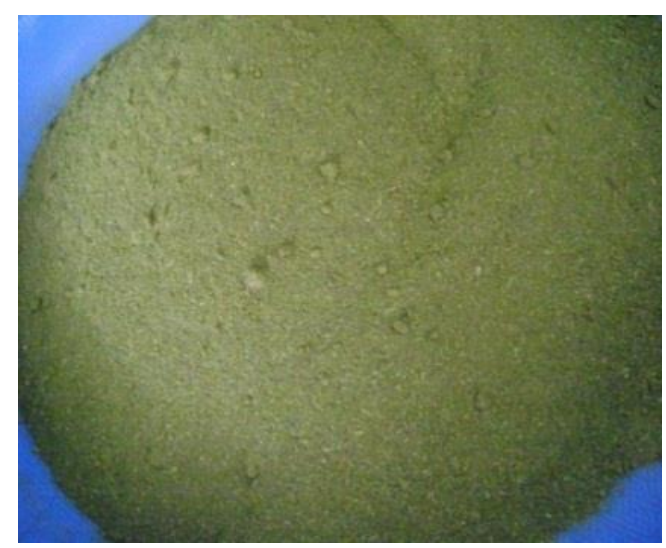

Photo 2 : Poudre de jeunes feuilles sèches de goyavier

\section{Poids vif}

Les effets de la supplémentation de l'aliment en poudre de feuilles de goyavier sur le poids vif des porcelets sont illustrés par les Figures 2A, 2B et 2C. Il en ressort que le poids vif des porcelets a augmenté régulièrement tout au long de l'essai, quel que soit le traitement. Indépendamment du sexe (Figure 2A), le poids vif a augmenté rapidement chez les porcelets nourris avec la ration contenant $1,5 \%$ de poudre de feuilles de Psidium guajava, aucune différence significative $(\mathrm{P}>0,05)$ n'a cependant été observée. Lorsqu'on considère le sexe, l'augmentation du poids vif a été plus importante chez les femelles ayant reçu la ration contenant $0,5 \%$ de poudre de feuilles de goyavier (Figure 2B). Par contre, chez les mâles (Figure 2C), ce sont les animaux traités au plus grand taux de poudre de feuilles de goyavier $(1,5 \%)$ qui ont présenté l'augmentation la plus rapide du poids vif. Au terme de l'essai (Figure 2) le niveau d'incorporation de la poudre de feuilles de Psiduim guajava, et le sexe des porcelets n'ont pas eu d'effets significatifs $(\mathrm{P}>0,05)$ sur le poids vif des porcelets. 


\section{Gain de poids}

L'évolution hebdomadaire du gain de poids des porcelets est illustrée par les Figures 3A, 3B et 3C. De manière générale, toutes les courbes de gain de poids ont la même tendance quel que soit le traitement où le sexe. Ainsi, le gain de poids a en général augmenté de la première à la troisième semaine de l'essai avant de baisser jusqu'à la fin de l'essai. Par contre, le traitement contenant $1,5 \%$ de poudre de feuilles de goyavier a induit le gain de poids significativement $(\mathrm{P}<0,05)$ plus élevé comparé au traitement contenant $1 \%$ de poudre de feuilles de goyavier. Indépendamment du sexe (Figure 3A), le gain de poids a augmenté plus rapidement chez les animaux recevant la ration contenant $1,5 \%$ de poudre de feuilles de goyavier du début à la troisième semaine de l'essai. Par contre, de la troisième semaine de traitement jusqu'à la fin de l'essai, ce sont les porcelets soumis à la ration contenant $0,5 \%$ de poudre de feuilles de goyavier qui ont gagné le plus rapidement du poids. Lorsque le sexe est considéré, les gains de poids les plus importants ont été observés chez les femelles soumises à la ration contenant $0,5 \%$ de poudre des feuilles de goyavier (Figure 3B). Chez les mâles (Figure $3 \mathrm{C}$ ), ce gain a été de manière générale plus important chez les porcelets ayant reçu la ration contenant $1,5 \%$ de poudre des feuilles de goyavier.

$\mathrm{Au}$ terme de l'essai, le niveau d'incorporation de la poudre de feuilles de Psidium guajava, et le sexe des porcelets n'ont pas eu d'effet significative $(\mathrm{P}<0,05)$ sur le gain de poids des porcelets.

A l'exception du lot dont la ration contenait $0,5 \%$ de poudre de feuilles de goyavier, le gain de poids des femelles a été non significativement $(\mathrm{P}>0,05)$ plus faible que celui des mâles quel que soit le traitement. Une corrélation positive et significative a été enregistrée entre le gain de poids et le poids vif des porcelets $(\mathrm{R}=+0,66 ; \mathrm{P}<0,01)$.

\section{Gain moyen quotidien}

L'évolution hebdomadaire du gain moyen quotidien des porcelets en fonction du niveau de poudre de feuilles de $P$. guajava dans l'aliment et du sexe est illustrée par les Figures 4A, 4B et 4C. Indépendamment du sexe (Figure $4 \mathrm{~A})$. Le gain moyen quotidien a augmenté significativement $(\mathrm{P}<0,05)$ chez les animaux recevant la ration contenant $0,5 \%$ de poudre de feuilles de $P$. guajava comparés à la ration contant $1 \%$ de cette poudre, du début à la troisième semaine de l'essai. Par contre, de la troisième semaine jusqu'à la fin de l'essai, c'est plutôt les porcelets soumis à la ration $0,5 \%$ de poudres qui ont eu un gain significatif $(\mathrm{P}<0,05)$ de poids. Lorsque le sexe est considéré, les gains moyens quotidiens les plus importants ont été observés chez les femelles soumises à la ration $\mathrm{T} 2$ contenant $0,5 \%$ de poudre des feuilles de goyavier (Figure 4B). Chez les mâles (Figure 4C), ce gain moyen quotidien a été de manière générale plus important chez les porcelets ayant reçu la ration contenant $1,5 \%$ de poudre des feuilles de goyavier. Au terme de l'essai, le niveau d'incorporation de la poudre de feuilles de Psiduim guajava, et le sexe des porcelets n'ont pas eu d'effet significative $(\mathrm{P}<0,05)$ sur le gain moyen quotidien des porcelets. A l'exception du lot dont la ration contenait à $0,5 \%$ de poudre des feuilles de goyavier, le gain moyen quotidien des femelles a été non significativement $(\mathrm{P}>0,05)$ plus faible que celui des mâles quel que soit le traitement. Des corrélations positives, fortes et significatives ont été observées respectivement entre le gain moyen quotidien et le poids vifs d'une part $(\mathrm{r}=+0,66 ; \mathrm{P}<0,01)$, puis le gain de poids d'autre part $(\mathrm{r}=+1 ; \mathrm{P}<0,01)$

\section{Indice de consommation}

La Figure 5 illustre l'évolution hebdomadaire de l'indice de consommation chez les porcelets nourris aux rations contenant 
la poudre des feuilles de goyavier. La baisse de l'indice de consommation a été plus faible du début à la troisième semaine de l'essai, puis il a augmenté rapidement de la troisième jusqu'à la fin de l'essai chez les porcelets recevant $1 \%$ de poudre de feuilles de goyavier. L'inverse a été observé chez les animaux qui ont reçu les rations contenant $0,5 \%$ et $1,5 \%$ de poudre de feuilles de goyavier, aucune différence significative $(\mathrm{P}>0,05) \quad$ n'a cependant été trouvée. Par ailleurs, des corrélations négativement fortes et significatives ont été observées respectivement entre l'indice de consommation et le poids vifs d'une part $(\mathrm{r}=-$ $0,65 ; \mathrm{P}<0,01)$, le gain de poids $(\mathrm{r}=-0,97$; $\mathrm{P}<0,01)$ et le gain moyen quotidien des porcelets $(\mathrm{r}=-0.97 ; \mathrm{P}<0,01)$.

\section{Efficacité alimentaire}

L'évolution hebdomadaire de l'efficacité alimentaire chez les porcelets nourris aux rations contenant la poudre des feuilles de goyavier est illustrée par la Figure 6. Il en ressort que l'efficacité alimentaire a augmenté du début à la troisième semaine de l'essai avant de diminuer fortement jusqu'à la fin de l'essai quel que soit le traitement. Au terme de l'essai, l'efficacité alimentaire a été significativement plus élevé chez les porcelets qui ont reçu la ration contenant $1,5 \%$ de poudre de feuilles de goyavier comparée à celle des animaux qui ont reçu $1 \%$ de cette poudre. Aucune autre différence significative n'a été enregistrée lorsqu'on compare les autres traitements. Des corrélations positives fortes et significatives ont été respectivement enregistrées entre l'efficacité alimentaire et le poids vifs $(\mathrm{r}=+0,66 ; \mathrm{P}<0,01)$, le gain de poids $(\mathrm{r}=+0,99 ; \mathrm{P}<0,01)$ et le gain moyen quotidien des porcelets $(\mathrm{r}=+0,99 ; \quad \mathrm{P}<0,01)$. Une corrélation négativement forte et significative a été observée entre l'efficacité alimentaire et l'indice de consommation des porcelets ( $\mathrm{r}=-$ $0.97 ; \mathrm{P}<0,01)$.

\section{Coût de production}

La Figure 7 matérialise l'évolution hebdomadaire du coût de production du $\mathrm{kg}$ de poids vif chez les porcelets nourris aux rations contenant la poudre des feuilles de goyavier. D'une manière générale, les courbes ont la même tendance. La baisse du coût de production a été plus faible du début à la troisième semaine de l'essai, puis il a augmenté rapidement de la troisième jusqu'à la fin de l'essai chez les porcelets recevant $1 \%$ de poudre de feuilles de goyavier, aucune différence significative $(\mathrm{P}>0,05)$ n'a été observée. Par ailleurs, une corrélation positive, forte, et significative a été trouvée entre le coût de production du $\mathrm{kg}$ de poids vifs et l'indice de consommation des porcelets ( $\mathrm{r}=+0.99$; $\mathrm{P}<0,01)$. De plus, des corrélations négatives faibles, moyennes, fortes et significatives ont été observées respectivement entre le coût de production et la consommation alimentaire $(\mathrm{r}=-$ $0.08 ; \mathrm{P}<0,01)$, le poids vif $(\mathrm{r}=-0.66 ; \mathrm{P}<0,01)$, le gain de poids $(\mathrm{r}=-0.97 ; \mathrm{P}<0,01)$, le gain moyen quotidien $(\mathrm{r}=-0.97 ; \mathrm{P}<0,01)$ et l'efficacité alimentaire des porcelets $(\mathrm{r}=-0.97$; $\mathrm{P}<0,01)$. 
Tableau 2 : Effet de la supplémentation de l'aliment en poudre des feuilles de goyavier et du sexe sur les performances de croissance et le coût de production chez les porcelets.

\begin{tabular}{|c|c|c|c|c|c|c|c|c|}
\hline \multirow{2}{*}{$\begin{array}{l}\text { Quantité de poudre } \\
\text { (\%) de } P \text {. guajava } \\
\text { dans la ration }\end{array}$} & \multicolumn{8}{|c|}{ Caractéristiques de la croissance et coût de production } \\
\hline & Sexe & $\begin{array}{l}\text { Consommation } \\
\text { alimentaire (kg) }\end{array}$ & $\begin{array}{c}\text { Poids vif } \\
\text { (kg) }\end{array}$ & $\begin{array}{c}\text { Gain de } \\
\text { poids (kg) }\end{array}$ & $\begin{array}{c}\text { Gain moyen } \\
\text { quotidien (g/j) }\end{array}$ & $\begin{array}{c}\text { Indice de } \\
\text { consommation }\end{array}$ & $\begin{array}{c}\text { Efficacité } \\
\text { alimentaire }\end{array}$ & $\begin{array}{l}\text { Coût de production } \\
\text { (FCFA) }\end{array}$ \\
\hline \multirow{3}{*}{$\mathbf{0}$} & $\delta^{\lambda}(n=3)$ & - & $19,33 \pm 2,52$ & $8,33 \pm 0,33$ & $297,62 \pm 11,90$ & - & - & - \\
\hline & $q(\mathrm{n}=3)$ & - & $18,67 \pm 1,55$ & $8,00 \pm 1,33$ & $285,71 \pm 47,62$ & - & - & - \\
\hline & $\delta+(n=6)$ & $34,01 \pm 4,55$ & $19,00 \pm 1,79$ & $8,17 \pm 1,17^{\mathrm{ab}}$ & $291,67 \pm 41,75^{\mathrm{ab}}$ & $2,86 \pm 0,39$ & $0,35 \pm 0,05^{\mathrm{ab}}$ & $753,25 \pm 103,20$ \\
\hline \multirow{3}{*}{0,5} & $\delta(n=3)$ & - & $19,00 \pm 1,00$ & $7,67 \pm 1,11$ & $273,81 \pm 39,68$ & - & - & - \\
\hline & $q(n=3)$ & - & $19,67 \pm 1,53$ & $9,33 \pm 0,88$ & $333,33 \pm 31,75$ & - & - & - \\
\hline & đo+ $(n=6)$ & $35,50 \pm 7,66$ & $19,33 \pm 1,21$ & $8,5 \pm 1,52^{\mathrm{ab}}$ & $303,57 \pm 54,16^{\mathrm{ab}}$ & $2,87 \pm 0,59$ & $0,36 \pm 0,06^{\mathrm{ab}}$ & $760,84 \pm 156,10$ \\
\hline \multirow{3}{*}{1} & $\delta(n=3)$ & - & $18,00 \pm 2,00$ & $7,00 \pm 0,67$ & $250,00 \pm 23,81$ & - & - & - \\
\hline & $q(n=3)$ & - & $17,33 \pm 2,52$ & $6,67 \pm 1,11$ & $238,10 \pm 39,68$ & - & - & - \\
\hline & $\delta^{\lambda}+(\mathrm{n}=6)$ & $35,50 \pm 7,66$ & $17,67 \pm 2,07$ & $6,83 \pm 1,75^{\mathrm{b}}$ & $244,05 \pm 41,75^{\mathrm{b}}$ & $3,51 \pm 0,67$ & $0,29 \pm 0,05^{\mathrm{b}}$ & $937,24 \pm 179,08$ \\
\hline \multirow{3}{*}{1,5} & $\hat{\sigma}(n=3)$ & - & $20,00 \pm 3,46$ & $9,00 \pm 2,00$ & $321,43 \pm 71,43$ & - & - & - \\
\hline & $q(n=3)$ & - & $19,33 \pm 2,31$ & $8,66 \pm 0,44$ & $309,52 \pm 15,87$ & - & - & - \\
\hline & $\delta^{\lambda}+(\mathrm{n}=6)$ & $35,50 \pm 4,87$ & $19,67 \pm 2,66$ & $8,83 \pm 1,72^{\mathrm{a}}$ & $315,48 \pm 61,51^{\mathrm{a}}$ & $2,78 \pm 0,63$ & $0,37 \pm 0,07^{\mathrm{a}}$ & $747,94 \pm 170,10$ \\
\hline
\end{tabular}




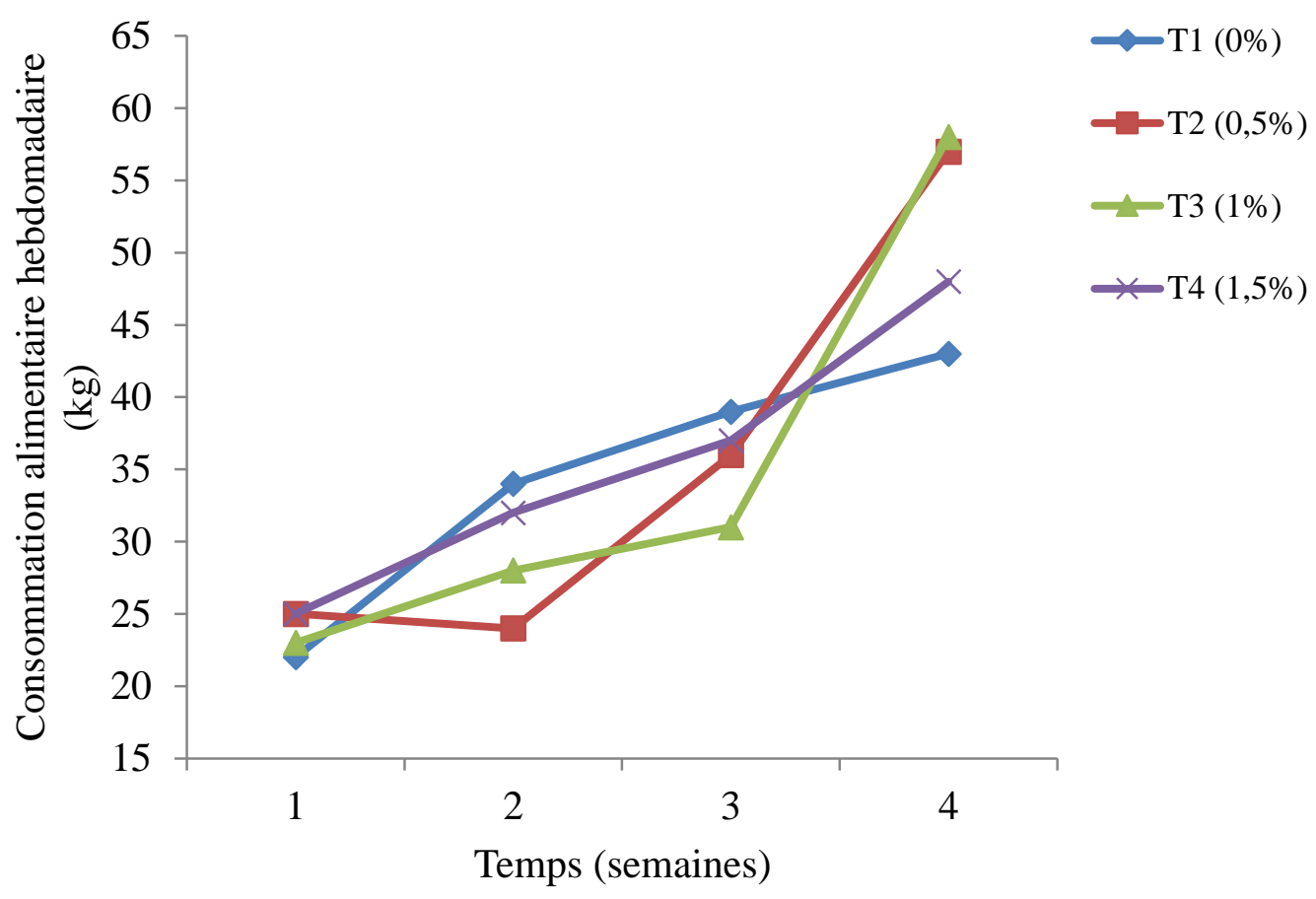

Figure 1 : Evolution hebdomadaire de la consommation alimentaire chez les porcelets soumis à différents niveaux de supplémentation de la poudre de feuilles de $P$. guajava dans l'aliment.

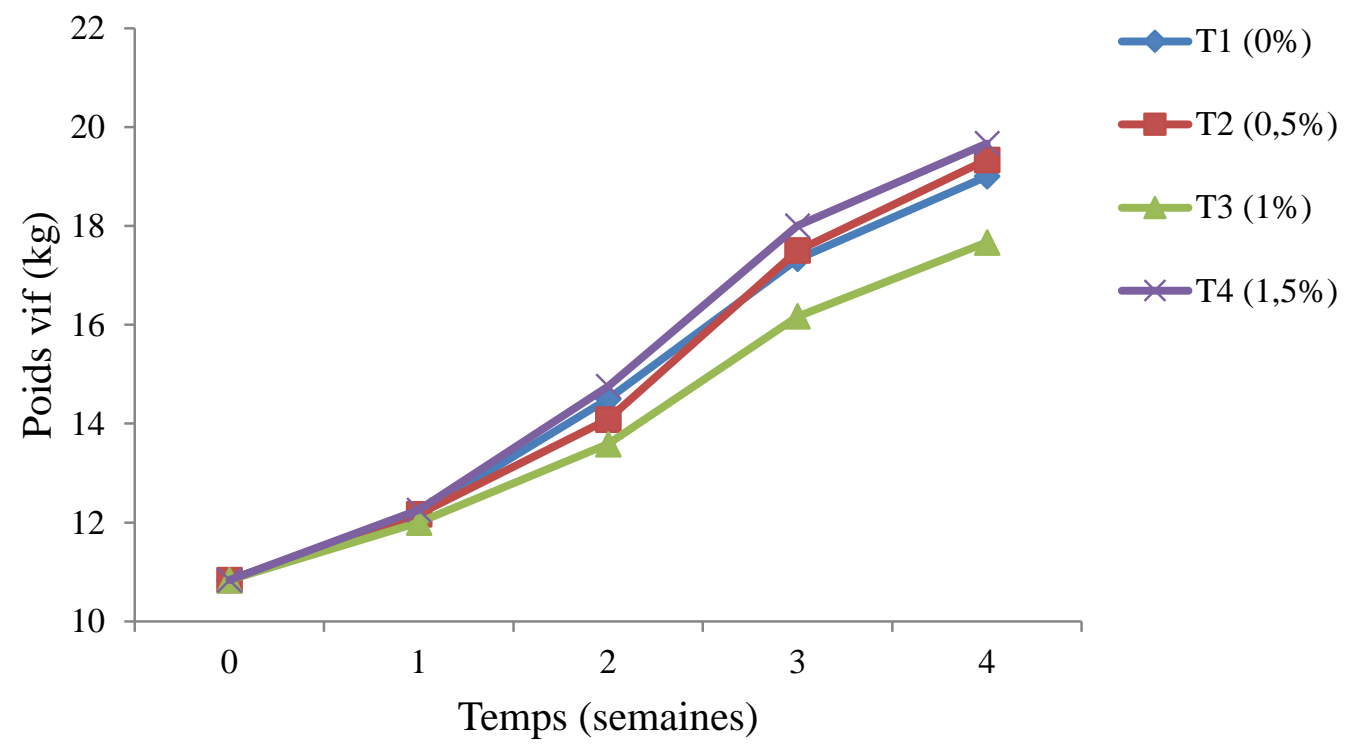

Figure 2A : Evolution hebdomadaire du poids vif chez les porcelets soumis à différents niveaux de supplémentation de la poudre de feuilles de $P$. guajava dans l'aliment. 
F. DJITIE KOUATCHO et al. / Int. J. Biol. Chem. Sci. 15(2): 635-651, 2021

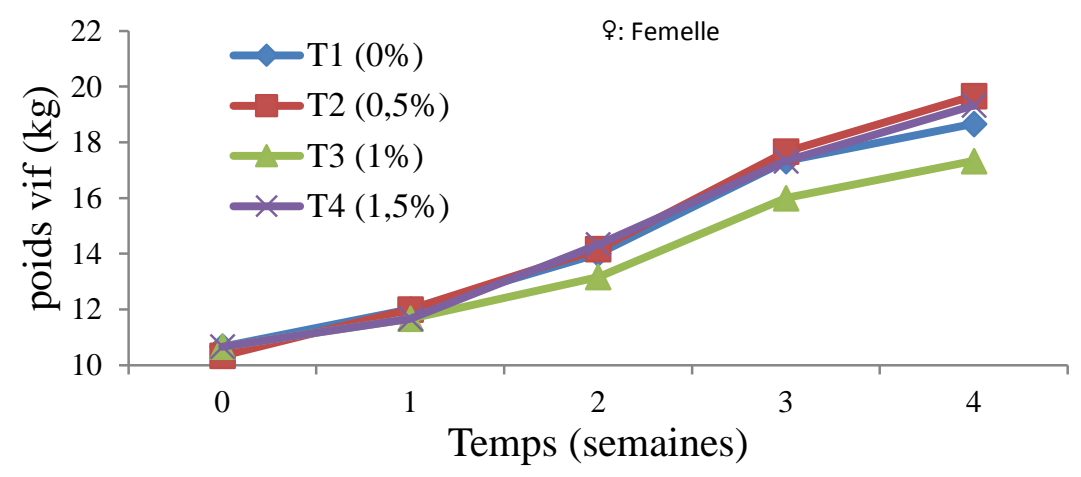

Figure 2B : Evolution hebdomadaire du poids vif chez les porcelets femelles soumis à différents niveaux de supplémentation de la poudre de feuilles de P. guajava dans l'aliment.

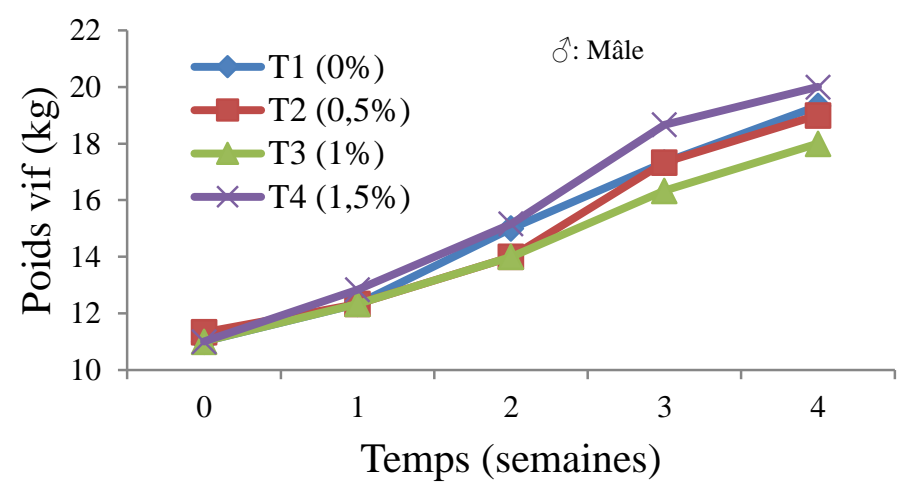

Figure 2C : Evolution hebdomadaire du poids vif chez les porcelets mâles soumis à différents niveaux de supplémentation de la poudre de feuilles de $P$. guajava dans l'aliment.

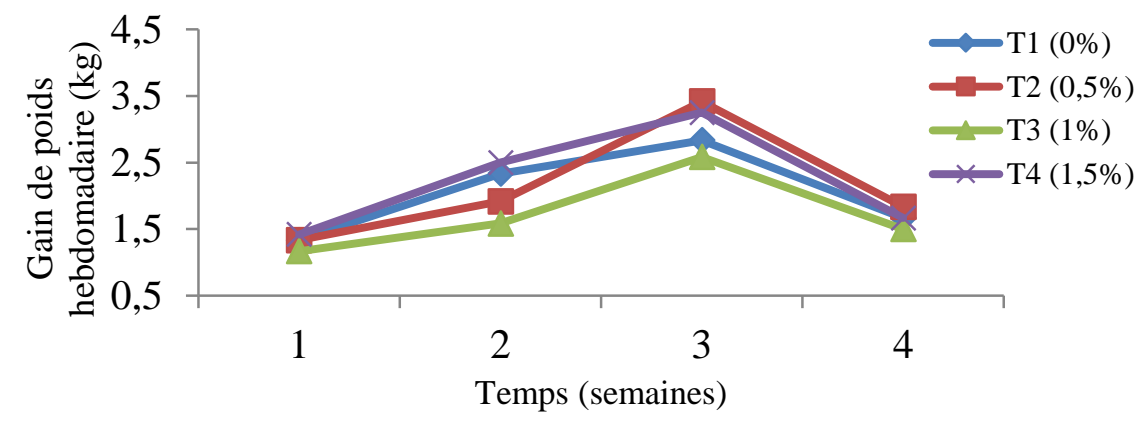

Figure 3A : Evolution hebdomadaire du gain de poids chez les porcelets soumis à différents niveaux de supplémentation de la poudre de poudre de feuilles de $P$. guajava dans l'aliment. 


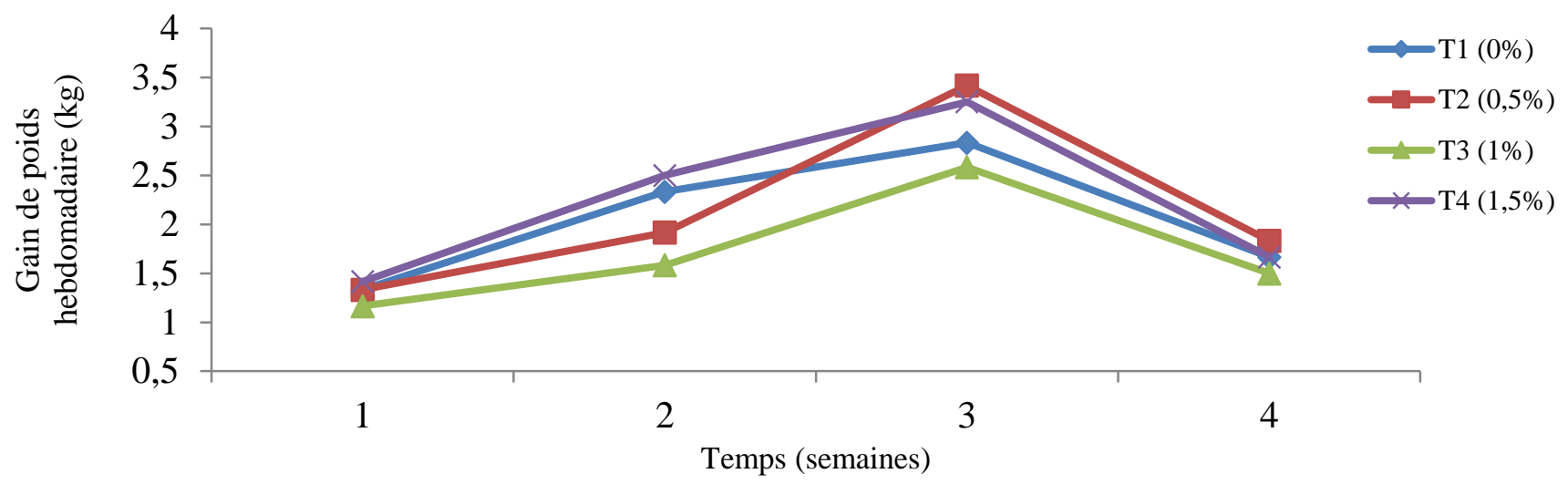

Figure 3A : Evolution hebdomadaire du gain de poids chez les porcelets soumis à différents niveaux de supplémentation de la poudre de poudre de feuilles de $P$. guajava dans l'aliment.

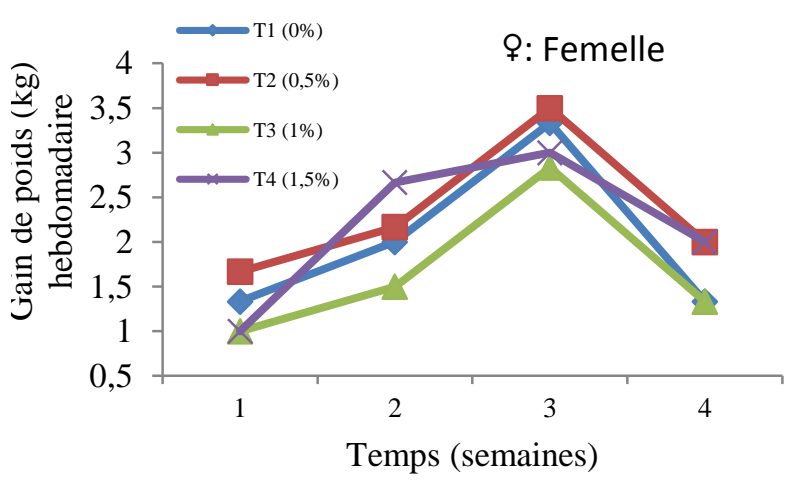

Figure 3B : Evolution hebdomadaire du gain de poids chez les porcelets femelles soumis à différents niveaux de supplémentation de la poudre de feuilles de P. guajava dans l'aliment.

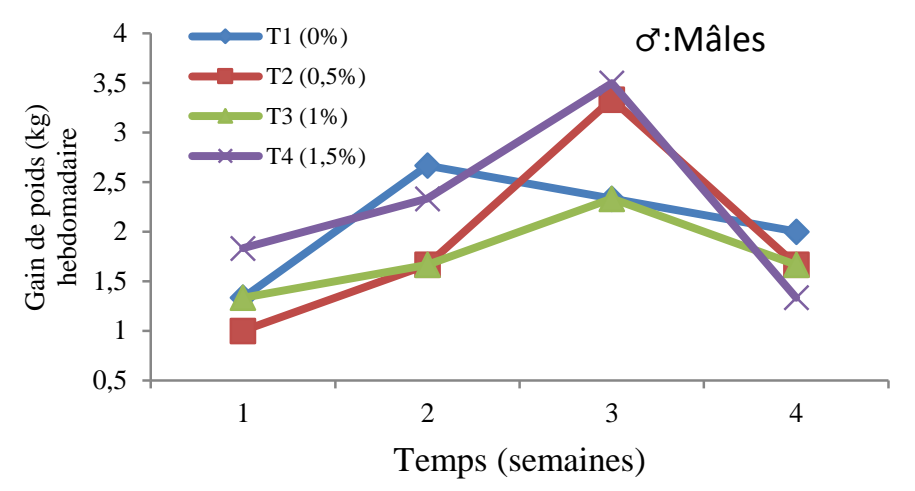

Figure 3C : Evolution hebdomadaire du gain de poids chez les porcelets mâles soumis à différents niveaux de supplémentation de la poudre de feuilles de $P$. guajava dans l'aliment. 


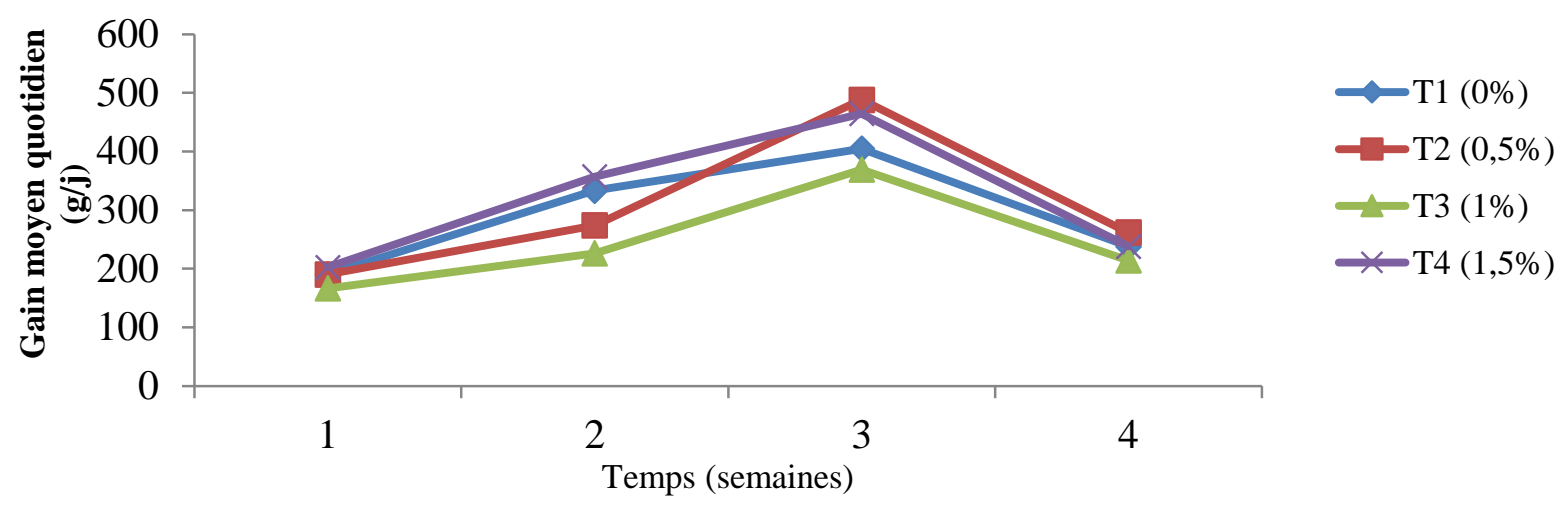

Figure 4A : Evolution hebdomadaire du gain moyen quotidien chez les porcelets soumis à différents niveaux de poudre de feuilles de $P$. guajava dans l'aliment.

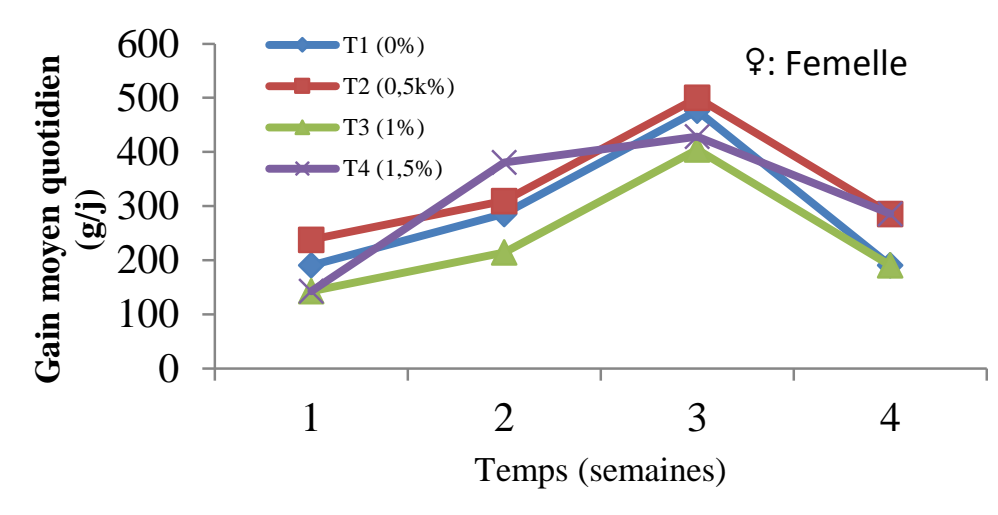

Figure 4B : Evolution hebdomadaire du gain moyen quotidien chez les porcelets femelles soumis à différents niveaux de poudre de feuilles de $P$. guajava dans l'aliment.

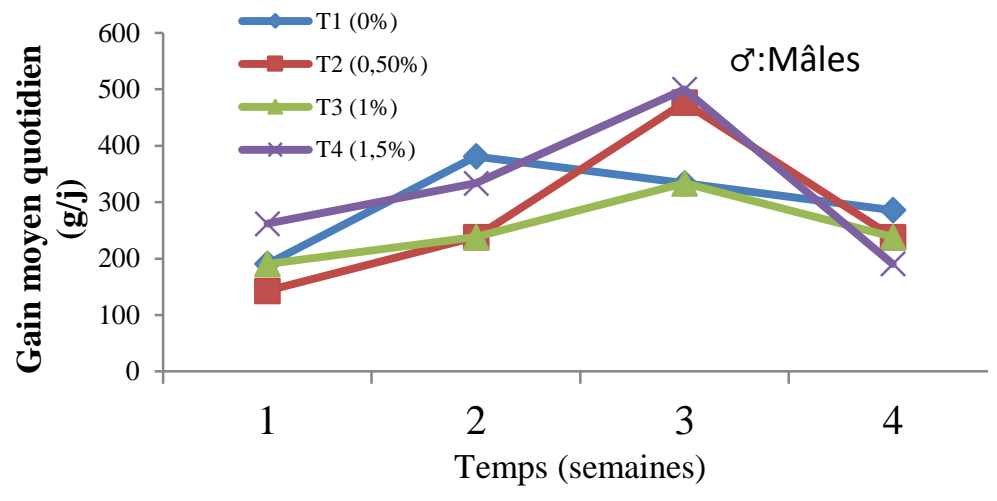

Figure 4C : Evolution hebdomadaire

du gain moyen quotidien chez les porcelets mâles soumis à différents niveaux de poudre de feuilles de $P$. guajava dans l'aliment. 


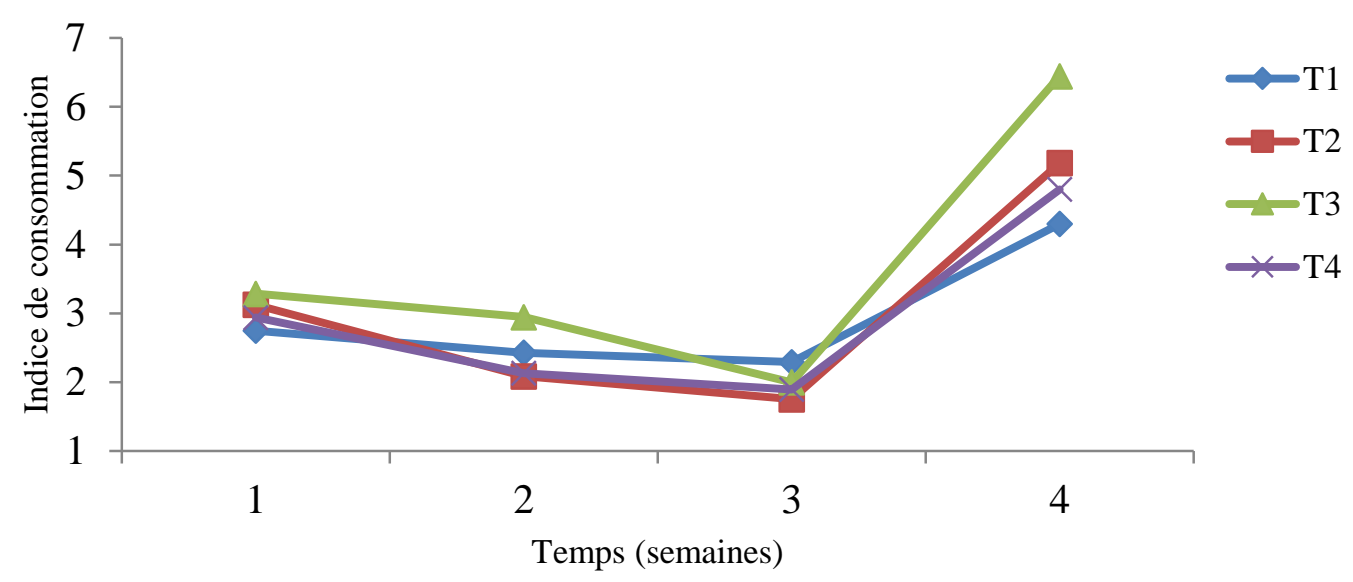

Figure 5 : Evolution hebdomadaire de l'indice de consommation chez les porcelets soumis à différents niveaux de supplémentation de la poudre de feuilles de $P$. guajava dans l'aliment.

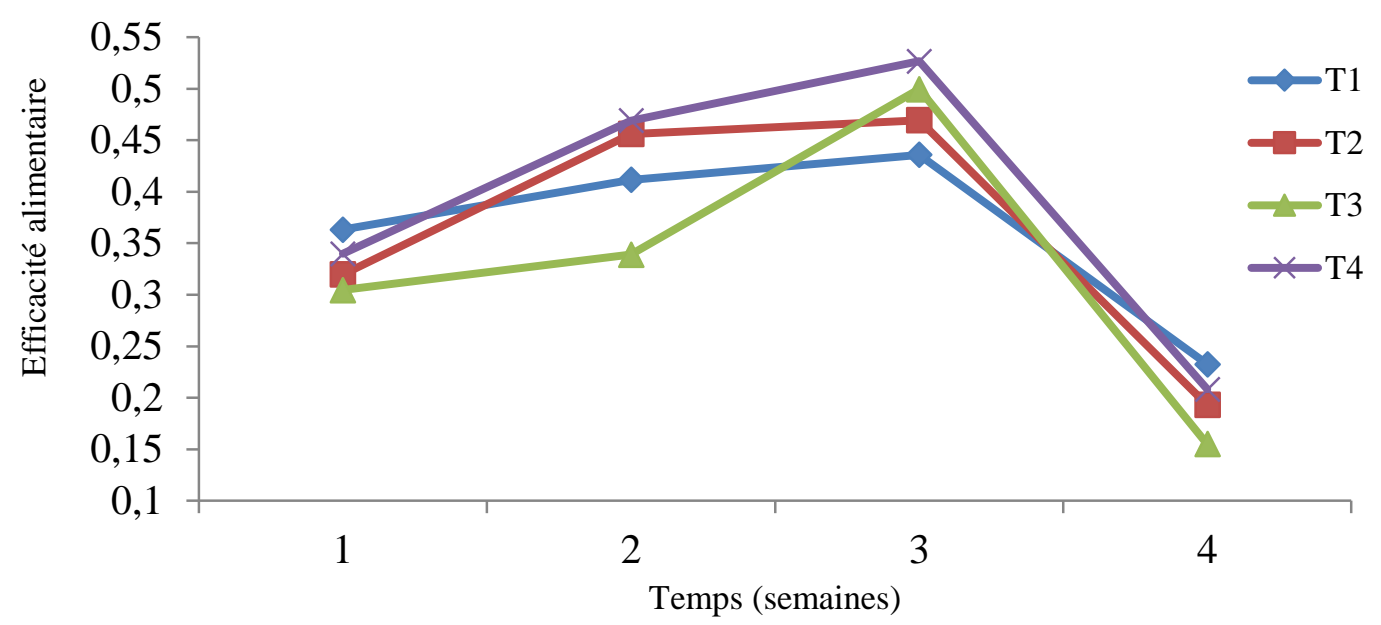

Figure 6 : Evolution hebdomadaire de l'efficacité alimentaire chez les porcelets soumis à différents niveaux de poudre de feuilles de $P$. guajava dans l'aliment.

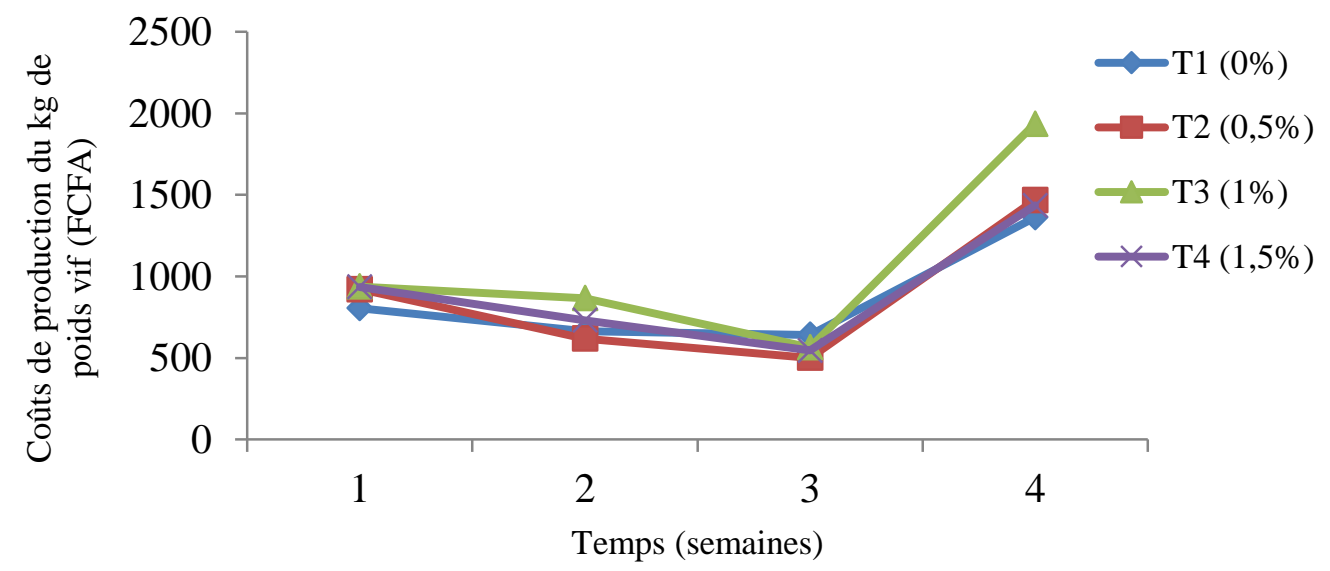

Figure 7 : Evolution hebdomadaire du coût alimentaire de production chez les porcelets soumis à différents niveaux de poudre de feuilles de $P$. guajava dans l'aliment. 


\section{DISCUSSION}

Les résultats obtenus dans la présente étude font apparaître que la poudre de feuilles de goyavier a entrainé une augmentation non significative de la consommation alimentaire. Ces résultats sont similaires à ceux de l'étude menée par (Zaminur et al., 2013) sur des poulets de chair. Ces derniers ont observé que l'incorporation des extraits de feuilles de goyavier n'affecte pas la prise alimentaire. Cette observation est contradictoire à l'idée déjà soutenue par Coto et al. (2008) et Wang et al. (2008) qui ont enregistré la baisse de la consommation alimentaire chez les poulets nourris respectivement avec des enzymes, des condiments, des probiotiques, des acides organiques et des minéraux. Cette augmentation non significative de la consommation alimentaire pourrait s'expliquer par l'action probable des composés se trouvant dans les feuilles de Psidium guayava qui stimuleraient le centre de la faim des porcelets, ou pourrait être due à l'action des composés contenus dans les feuilles qui amélioraient la saveur et l'odorat de l'aliment. Les données sur le poids vif ont montré une augmentation non significative du poids des porcelets dont l'aliment renfermait $1,5 \%$ de poudre des feuilles de goyavier. Ces résultats sont en contradiction avec ceux trouvés par Rao et al. (2004) chez le porc traité avec les extraits des feuilles de goyavier, Zaminur et al. (2013) chez les poulets de chair traités avec les extraits des feuilles de goyavier, Mahmoud et al. (2013) chez les poulets de chair traité avec les extraits des feuilles de goyavier et Nouboudem (2014) chez le cobaye traité avec les huiles essentielles des feuilles de goyavier entrainent une augmentation du poids vif chez les animaux. Cette augmentation non significative du poids vif final serait la conséquence de celle de la consommation alimentaire observée dans les lots traités. En effet, l'augmentation du poids vif résulte d'une accumulation des nutriments issus de l'aliment consommé. Par ailleurs, cette augmentation est sans doute imputable aux propriétés androgéniques que possèdent les feuilles de goyavier. En effet, les feuilles de goyavier renferment les flavonoïdes et les phénols qui grâce à leurs effets androgéniques sont susceptibles d'agir sur la croissance des tissus à développement rapide et de stimuler la synthèse des protéines du muscle. La présente étude a également révélé une augmentation significative du gain de poids des porcelets traités avec la ration contenant $1,5 \%$ de poudre de feuilles de goyavier comparé au lot témoin. Ces résultats corroborent ceux trouvés par Leonard et al. (2011) chez des truies recevant via l'aliment $10 \mathrm{~g} /$ jour d'extrait d'algue au $109^{\text {ème }}$ jour de gestation, Woode et al. (2011) chez les rats mâles traités aux extraits éthanoliques des fruits de Xylopia aethiopica aux doses 30, 100 et $300 \mathrm{mg} / \mathrm{kg} \mathrm{pc}$; Ekaluo et al. (2013), chez les rats traités aux extraits aqueux de feuilles de Psidium guajava (0, 100, 200 and 300 mg/kg pc) et Farouk et al. (2013) chez les rats mâles traités à l'huile essentielle de Syzygium aromaticum $(0,1 \mathrm{mg} / \mathrm{kg} \mathrm{pc})$. Cette augmentation significative s'expliquerait par celle du poids vif enregistrée dans ce lot. Une corrélation positive, forte et hautement significative a d'ailleurs été trouvée entre ces deux caractéristiques. Cela pourrait aussi s'expliquer par la présence de certains composés comme les tanins, les flavonoïdes et les phénols qui auraient détruit les microorganismes pathogènes par leurs activités bactéricides et antifongiques, tout en favorisant le développement des microorganismes bénéfiques. Elle traduirait aussi l'effet positif des flavonoïdes contenus dans la poudre de feuilles $P$. guajava utilisés qui aurait facilité l'oxydation des biomolécules, la digestion, l'absorption et l'utilisation efficace des nutriments.

De nos résultats, il ressort que la poudre des feuilles de goyavier a amélioré de manière non significative l'indice de consommation. Ces résultats sont contradictoires à ceux de Murry et al. (2006), Al-Harthi (2006), AbdelFattah et al. (2008), Wang et al. (2008), Idoui 
et al. (2009) chez les poulets de chair nourris aux additifs alimentaires tels que les enzymes, les condiments, les probiotiques et les acides organiques qui améliorent respectivement l'indice de consommation et l'efficacité alimentaire par rapport au témoin. Dans le cadre de notre étude, l'écart entre l'indice de consommation des différents traitements est imputable à un meilleur gain de poids. D'ailleurs des corrélations négatives et fortes ont été enregistrées entre l'indice de consommation et le gain de poids. En effet, suite aux effets bénéfiques des flavonoïdes, des phénols et aux tannins de la poudre de feuilles de goyavier qui ont modifié le microbiote du tube digestif, favorisé la digestion et l'utilisation efficace des nutriments; d'où l'efficacité alimentaire la plus élevée trouvée chez les porcelets du lot traité avec $1,5 \mathrm{~kg}$ de poudre de feuilles de goyavier.

Nos résultats montrent que le coût de production le plus faible a été obtenu dans le lot traité avec la dose la plus élevée $(1,5 \%)$ de poudre de feuilles de goyavier. Ceci pourrait être expliqué par les propriétés bioactives de la plante qui auraient favorisé la transformation de l'aliment ingéré en protéines animales. Ces résultats corroborent ceux de Ferket et al. (2002) qui ont montré que le coût de production du $\mathrm{kg}$ de poids vif baisse avec le taux d'incorporation du grenadier dans l'aliment des poulets de chair.

\section{Conclusion}

$\mathrm{Au}$ terme de cette étude portant sur l'effet de la supplémentation de l'aliment en poudre des feuilles de goyavier (Psidium guajava) sur les performances de croissance des porcelets, il a été conclu que la poudre des feuilles de goyavier n'a pas eu d'effet significatif sur la consommation alimentaire, le poids vif et l'indice de consommation;la poudre des feuilles de goyavier a entrainé chez les sujets traités à $1,5 \%$ un meilleur gain de poids, gain moyen quotidien et l'efficacité alimentaire chez les porcelets et le coût de production a été meilleur chez ceux traités à $1,5 \%$ de poudre des feuilles de goyavier. Au regard de ce qui précède, sur le plan zootechnique, la ration contenant $1,5 \%$ de la poudre de feuilles de goyavier semble être la plus indiquée bien que sur le plan économique, il induit un coût de production comparable à celui du traitement ne recevant pas de poudre de feuilles de goyavier.

\section{CONFLIT D'INTERETS}

Les auteurs de cet article certifient qu'il ne fait l'objet d'aucun conflit d'intérêts.

\section{CONTRIBUTIONS DES AUTEURS}

FN a conçu et mis en place le dispositif expérimental, a coordonné et orienté la réalisation technique et scientifique du travail ; VMKS a réalisé des essais et a collecté des données ; FDK a réalisé l'analyse statistique et a participé à la rédaction du manuscrit ; tous les auteurs ont lu et approuvé cette version du travail.

\section{REMERCIEMENTS}

Les auteurs remercient $M$. Nkenlifack Gislain Victor, Directeur général de NGVTRACO Cameroun pour avoir accepté la conduite des essais dans son entreprise, ainsi que le personnel pour leur accueil chaleureux et leur collaboration.

\section{REFERENCES}

Abdel-Fattah SA, El-Sanhoury MH, ElMednay NM, Abdel-Azeem F. 2008. Thyroid activity, some blood constituents, organs morphology and performance of broiler chicks fed supplemental organic acids. International Journal of Poultry Science, 7(3): 215222. DOI: $10.3923 /$ ijps.2008.215.222

Al-Harthi. 2006. Impact of supplemental Feed Enzymes, Condiments mixture or Their Combination on Broiler Performance, nutrients Digestibility and Plasma Constituents. International Journal of 
Poultry Science, 5(8): 764-771. DOI: 10.3923/ijps.2006.764.771

Chen Y, Yen GC. 2007. Antioxidant activity and free radical-scavenging capacity of extracts from guava (Psidium guajava L.) leaves. Food Chemistry, 101: 686-694. https://doi.org/10.1016/j.foodchem.2006. 02.047

Coto CF, Yan F, Cerrate S, Wan Z, Sacakli P, Halley JT, Wiernusz CJ, Martinez A, Waldroup PW. 2008. Effect of dietary level of calcium and non-phytate phosphorus in broiler starter diets on live performance, bone development and growth plate conditions in male chick fed a corn-based diet. International Journal of Poultry Science, 7(7): 638-645. DOI:10.3923/IJPS.2008.101.109

Delesalle CFV. 2016. Ethnopharmacognosie vétérinaire en élevage Avicole, Bovin, Bubalin et porcin au Cambodge Thèse de Medecine vétérinaire. Ecole Nationale Vétérinaire D’alfort, Faculté de Médecine de Créteil, France 315p.

Ekaluo B, Erem A, Omeje S, Ikpeme V, Ibiang YB, Ekanem E. 2013. Aqueous leaf extract of guava: a non-toxic male fertility booster. Journal of Environmental Science, Toxicology and Food Technology, 3(2): 21-23.

Farouk B, Abdelkrim B, Malika BS, Badreddine AK, Djallel EH, Nasreddine T. 2013. Ameliorative effects of Syzygium aromaticum essential oil on fertility in male rats exposed to manganese. Advances in Sexual Medicine, $\quad 3(4)$ : $\quad 85-91$. DOI: $10.4236 /$ asm.2013.34013

Ferket PR, Parks CW, Grimes J. 2002. Benefits of dietary antibiotic and mannanoligosaccharide supplementation for poultry. Department of Poultry Science. North Carolina State University.

Ferket PR, Van Heugten E, Van Kempen TATG, Angel R. 2002. Nutritional strategies to reduce environmental emissions from non-ruminants 1,2 . Journal of Animal Sciences, 80: 168-182. https://doi.org/10.2527/animalsci2002.80 E-Suppl_2E168x

Idoui T, Boudjerda D, Leghouchi E, Karam N. 2009. Activité probiotique de lactobacillus plantarum : étude réalisée chez le poulet de chair ISA 15. Huitièmes Journées de la Recherche Avicole, St Malo. 25 et 26 Mars.

Lessard Martin 2004. Utilisation des probiotiques chez le porc-modulateurs potentiels de la santé intestinale. Colloque sur la production porcine. CRAAO. $14 \mathrm{p}$.

Machebe NS, Agbo CU, Onuaguluchi CC. 2011.Oraladministration of Gongronema latifolia leaf meal: Implications on carcass and haematological profile of broiler finishers raised in the humid tropics. African Journal of Biotechnology, 10: 5800-5805. DOI: 10.5897/AJB11.074

Mahmoud R, El-Sayed, Ibrahim, Doaa, Badawi, El-Sayed M. 2013. Effect of supplementation of broiler diets with guava leaves and/or olive oil on growth, meat composition, blood metabolites and immune response. Benha Veterinary Medical Journal, 2(55): 23-32.

Motsa'a, Sob J, Defang HF, Tiambo Keambou C. 2019. Socio-Economic and Technical Characteristics of Pig (Sus Scrofa Domesticus) Production System in the Humid Forest with Monomodal Rainfall Agroecological Zone of Cameroon. International Journal of Biological and Chemical Sciences, 12(5): 2318. DOI: https://doi.org/10.4314/ijbcs.v12i5.31.

Murry AC, Hinton A, Buhr. 2006. Effect of Botanical Probiotic Contening Lactobacilli on Growth Performance and Populations of Bacteria in the Ceca, cloaca, and Carcass Rinse of Broiler Chickens. International Journal of Poultry Science, 5(4): 344-350. DOI: $10.3923 /$ ijps.2006.344.350 
Mweugang NN, Tendonkeng F, Miégoué E, Matumuini FEN, Zougou GT, Fonteh FA, Boukila B, Pamo ET, 2016. Effets de l'inclusion de feuilles de manioc (Manihot esculenta Crantz) dans la ration sur les performances de reproduction du cobaye (Cavia porcellus L.) local camerounais Int. J. Biol. Chem. Sci., 10(1): 269-280. DOI: 10.4314/ijbcs.v10i1.21

N'Guessan-Irié AGN, Kablan BJ, SiransyKouakou NG, Konan A, Datté YJ. 2012. Propriétés bioactives de l'extrait aqueux des feuilles de Psidium guajava (Myrtaceae). CAMES, Pharmacopée et Médecine Traditionnelle Africaine, 16(2012) : 16p.

Nikolova M, Grigorova S, Abadjieva D, Penkov D. 2010. Investigation of the effect of Tribulus terrestris extract on some characteristics of the reproductive capacity of guinea fowl. Biotechnology of Animal Husbandary; 26: 259-66. DOI: https://doi.org/10.2298/BAH10042 $59 \mathrm{~N}$

Nouboudem CS. 2014. Effets de l'huile essentielle des feuilles de goyavier (Psidium Guajava) sur quelques paramètres de reproduction et sanguins chez le cobaye. Thèse de Master of science. FASA. UDs. 63p.

Nwangwa EK, Mordi J, Ebeye OA, Ojieh AE. 2007. Testiculat regenerative effects induced by extracts of Telfairia occidentalis in rats. Caderno de Pesquisa Serie Biol., 19: 27-35.

Oh WK, Lee CH, Lee MS, Bae EY, Sohn CB. 2005. Antidiabetic effects of extracts from Psidium guajava. Journal of Ethnopharmacology. 93:11-415 DOI: 10.1016/j.jep.2004.09.041

Pousset J-L.1989. Plantes Médicinales Africaines. Utilisation Pratique (Tome I). Editions Marketing: Paris; 230p.

Rao TM, Ravia, Rao DS, Prasad JR, Roddy PS, Rao ZP. 2004. Effect of inclusion guava pomace in pig diets on growths performance, nutrient utilization of carcass characteristics. Anim. Nut, Teed Technology, 4(1): 43-52.

Robineau-Germosén L, Weniger B, Carballo A, Lagos-Witte S. 1999. Pharmacopée Végétale Caribéenne (Première édition). Édition Désormeaux ; 493p.

Wang Z, Cerrate S, Yan F, Sacakil P, Waldroup PW. 2008. Comparison of different concentrations of inorganic trace minerals in broiler diets on live performance and mineral excretion. International Journal of Poultry Science, 7(7): $\quad 625-629 . \quad$ DOI: 10.3923/ijps.2008.625.629

Woode E, Alhassan A, Chrissie S, Abaidoo. 2011. Effect of ethanolic fruit extract of Xylopia aethiopica on reproductive function of male rats. International Journal of Pharmacology and Biomedical Research, 2(3): 161-165.

Zaminur R, Siddiqui MN, Khatum MA, Kam Ruzzamun M. 2013. Effect of guava leaf (Psidium guajava) meal on production performances antimicrobial sensitivity in commercial broiler. Journal of Natural Products, 6: 177-187. 